\title{
REAL-TIME SOIL SENSING WITH NIR SPECTROSCOPY
}

\author{
Jianying Sun, Minzan Li, Lihua Zheng, Ning Tang \\ Key Laboratory of Modern Precision Agriculture System Integration Research, China \\ Agricultural University, Ministry of Education, Beijing 100083, China \\ Corresponding author,Email: limz@cau.edu.cn
}

\begin{abstract}
The grey-brown alluvial soil is a typical soil in the Northern China. It was selected as research object to reveal feasibility and possibility of real-time analyzing soil parameter with NIR spectroscopic techniques. 150 samples were collected from a winter wheat farm. And then the NIR absorbance spectra were rapidly measured under the original conditions by a Nicolet Antaris FT-NIR analyzer. Three soil parameters, soil moisture, SOM, TN content, were analyzed. For soil moisture content, a linear regression model was available, using $1920 \mathrm{~nm}$ of wavelength with correlation coefficient of 0.937 , So that the results obtained could be directly used to real time evaluate soil moisture. SOM content and TN content were estimated with a multiple linear regression model, $1870 \mathrm{~nm}$ and $1378 \mathrm{~nm}$ wavelengths were selected in the SOM estimated model, while $2262 \mathrm{~nm}$ and $1888 \mathrm{~nm}$ wavelengths were selected in the TN estimated model. The results showed that soil SOM and TN content could be evaluated by using NIR absorbance spectra of soil samples.
\end{abstract}

Keywords: NIR spectroscopy, chemometrics, soil moisture, soil organic matter, soil total nitrogen

\section{INTRODUCTION}

Describing the variability of soil parameters is an important step for promoting precision agriculture. So it is necessary to look for a real-time evaluating method of soil parameters.

NIR spectroscopy appears as a rapid, convenient and simple nondestructive technique that can be used to quantify several soil properties

Sun, J., Li, M., Zheng, L. and Tang, N., 2008, in IFIP International Federation for Information Processing, Volume 259; Computer and Computing Technologies in Agriculture, Vol. 2; Daoliang Li; (Boston: Springer), pp. 1403-1406. 
in many researches (Shimano T.). Li Minzan, A. Sasao, and S. Shibusawa have taken Kanto Loam as the research object and developed some estimation models of soil parameters based on spectral reflectance of raw soil samples (Li M.Z. 2000). In addition, research showed that NIR spectral of soil was largely affected by soil type. Thus, the grey-brown alluvial soil in the Northern China was selected as research object, and the estimation models of soil moisture content, SOM, and TN were developed with NIR spectra of raw soil samples.

\section{MATERIALS AND METHODS}

Soil samples were collected from an experimental farm of winter wheat in China Agricultural University. After the soil samples were taken into the lab, NIR absorbance spectra and soil moisture were rapidly measured under their original conditions, The NIR absorbance spectra were measured by a Nicolet Antaris FT-NIR analyzer. Then each soil sample was divided into two parts. One was used to directly analyze soil moisture. The other was used to analyze soil parameters after air-dried. Moisture was measured by 1050C$24 \mathrm{~h}$ method with an electric fan heater. Air-dried soil samples were analyzed using chemistry method. The soil parameters analyzed were soil organic matter (SOM), soil total nitrogen (TN), soil total phosphorus, soil total potassium and soil $\mathrm{pH}$.

\section{RESULT AND DISCUSSION}

\subsection{Moisture}

The soil moisture content has high correlation with original absorbance at every waveband. 90 data sets were used as calibration group to build an estimation model, and the other 60 data sets were used as validation group to check the model. Using absorbance at $1920 \mathrm{~nm}$, the wavelength with highest absorbance peak, a single linear regression model was established.

$$
y=17.706-2.167 x_{1920}
$$

where: $x_{i}$ is the original absorbance at wavelength of $\mathrm{i}$;

$\mathrm{y}$ is the soil moisture content

The correlation coefficient of the regression model is (Rc2)0.936, F and $t$ test showed that the characteristics of the model and the regression 
parameters were significant. The results of the calibration and validation of the model is shown in Figure 1:

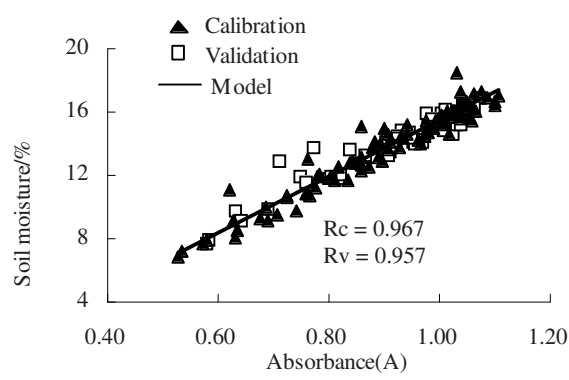

Figure 1. Calibration and validation of soil moisture SLR model



Figure 2. Calibration and validation of TN PLSR Model

\section{$3.2 \quad \mathrm{TN}$}

Four wavelengths were selected form the wavebands with higher correlation coefficient, which were $2240 \mathrm{~nm}, 2090 \mathrm{~nm}, 1901 \mathrm{~nm}$ and $1827 \mathrm{~nm}$. Using all four data sets a multiple linear regression was executed. In order to eliminate the effect of multicollinearity, PLSR was carried out. Using Leave One Out - Cross Validation, the PLS regression was conducted. The model was as follows:

$$
y=0.237-2.492 x_{2240}+0.263 x_{2090}+6.138 x_{1901}-4.285 x_{1827}
$$

where: $x_{i}$ is the NIR absorbance at wavelength of $\mathrm{i}$; $\mathrm{y}$ is the soil TN content

The result of calibration and validation is shown in Figure 2. The correlation coefficient of the model (RC2) was 0.601, and the correlation coefficient of validation (RV2) was 0.643 . In addition, it would be observed that there were non-linear factors in the model from Figure 2.

\subsection{SOM}

Difference of soil SOM content is also very little, and the correlation coefficient between soil SOM content and the original absorbance spectra or its first spectra derivation was very low. The maximum is lower than 0.4. Regression model was established using 30 new data preprocessed from original NIR absorbance spectra. The spectral variable used was the first derivative of NIR absorbance. Two wavelengths were selected based on correlation analysis, which are $1870 \mathrm{~nm}$ and $1038 \mathrm{~nm}$. Using the above two wavelengths a multiple linear regression model was established as follows: 


$$
y=1.195-515.471 x_{1870}+159.268 x_{1038}
$$

where: $x_{i}$ is the first spectral derivation at wavelength of $\mathrm{i}$; $\mathrm{y}$ is the SOM content

The correlation coefficient of the model (RC2) is 0.607 . The significance of $F$ test was $3.423 \times 10-6$ and passed the F test, which implied that the NIR absorbance at the above two wavelengths had notable linear correlation with SOM content. The P-value of t test respectively was 0.0005, 0.0006 and 0.0037 respectively, and passed the $t$ test. The preprocessed new 15 data sets were used as validation data sets, and the correlation coefficient of validation reached to (RV2) 0.711.

\section{CONCLUSION}

The soil moisture content has high correlation with NIR original absorbance. It was feasible evaluating soil TN and SOM content with NIR absorbance spectra of raw soil samples, and the regression model obtained from the first deviation data was more feasible since the deviation spectra could eliminate noises. And the results obtained could be taken as theoretic basis for the analysis of real-time soil parameters. In order to further improve precision of model, it is necessary to intentionally implement soil nutritionstress in next experiment, and developed a more feasible evaluation model.

\section{ACKNOWLEDGEMENTS}

This study was supported by National 863 Program (2006AA10A301).

\section{REFERENCES}

Li M.Z., Sasao A., Shibusawa S., et al. Soil parameters estimation with NIR spectroscopy. Journal of the Japanese Society of Agricultural Machinery, 2000, Vol. 62(3), pp. 111-120

Li N., Min S.G., Tan F.L., Zhang M.X., Ye S.F. Nondestructive Analysis of Protein and Fat in Whole-kernel Soybean by NIR, Spectroscopy and Spectral Analysis, 2004, Vol. 24(1), pp. 45-49

Shimano T. New design of geodesic lenses. MOC/GRIN'89, Tokyo: 130-135 